\title{
IL-23 tees off enthesitis
}

The interleukin-23 (IL-23) signalling pathway has previously been linked with spondyloarthritis, but it has been unclear how this cytokine can promote disease pathogenesis. A new research study published in Nature Medicine has shown that, in mice, IL-23 promotes a pathology that resembles spondyloarthritis by acting on a previously unidentified subset of T cells that reside in the enthesis (the junction between tendon and bone).

A link between IL-23 and spondyloarthritis had already been established, as polymorphisms in the IL-23 receptor are associated with ankylosing spondylitis and increased serum levels of IL-23 are found in patients with this disease. However, the exact role of IL-23 in spondyloarthritis was unclear. Sherlock et al. investigated whether IL-23 was involved in the development of enthesitis, a key feature of spondyloarthritis, by using a passive transfer model of arthritis that is induced by injecting mice with antibodies specific for type II collagen. The researchers showed that the development of enthesitis in this model was dependent on IL-23, as blocking IL-23 during the induction of arthritis reduced both the clinical disease scores and the levels of entheseal inflammation in the mice. Importantly, they found that IL-23 expression alone was sufficient for inducing enthesitis.
Indeed, transgenic mice overexpressing IL-23 developed enthesitis and other disease features reminiscent of human spondyloarthritis, including new entheseal bone formation and inflammation at the aortic root and valve.

Exploring the mechanisms involved, the authors showed that entheses contain a resident population of innate-like $T$ cells that express the IL-23 receptor and CD3, but not CD4 or CD8. Further characterization of these resident $T$ cells showed that they express the transcription factor ROR $\gamma t$ and produce IL-17 and IL-22 in response to IL-23 stimulation. IL-17 and IL-22 are cytokines that have also been implicated in the pathogenesis of spondyloarthritis, and IL-23-transgenic mice that were treated with blocking antibodies specific for IL-17 and IL-22 had reduced clinical disease scores. Overexpression of IL-22 in mice promoted bone remodelling and increased footpad swelling, and IL-22 was found to induce STAT3 (signal transducer and activator of transcription 3) phosphorylation in osteoblasts in vitro. The authors suggest that IL-23 may be important for promoting the upregulation of pro-inflammatory gene expression in the enthesis, whereas IL-22 is more important for inducing bone remodelling.

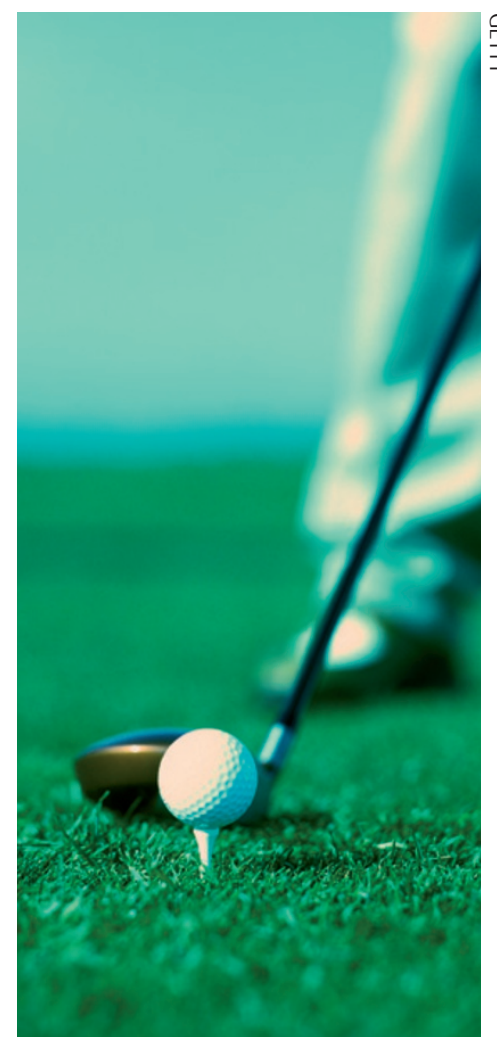

These data provide a vital insight into the immunopathogenesis of spondyloarthritis and identify the innate-like entheseal T cells as a future therapeutic target.

Katrina Ray, Associate Editor, Nature Reviews Rheumatology This article is modified from the original in Nature Rev. Rheumatol. (doi:10.1038/nrrheum.2012.117)

\section{reside in the} enthesis

昌

